\title{
P 33 SAFETY AND CLINICAL EFFECTIVENESS OF TRICLOFOS FOR PROCEDURAL SEDATION IN CHILDREN: A SYSTEMATIC REVIEW
}

Helen Sammons, Imti Choonara, Badriyah Alotaibi. University of Nottingham

10.1136/archdischild-2015-308634.41

Aim To evaluate the efficacy and safety (adverse events (AEs)) of triclofos for procedural sedation in children.

Methods MEDLINE (1948-Jan 2012), EMBASE (1980-Jan 2012), COCHRANE (1974-Jan 2012) and CINAHL (1974-Jan 2012) were searched using combinations of the following search terms: "triclofos" and "children or infant or pe"diatric" or neonate or adolescence or adolescences or adolescent" and "sedation". The search was limited to the studies that assessed triclofos safety and/or efficacy in children up to 18 years, undergoing procedural sedation. Trial quality was assessed using the Jadad score for RCTs and STROBE scoring checklist for observational studies.

Results Of the 17 articles identified, 12 studies evaluated the use of triclofos for painless procedural sedation and five for painful procedural sedation. Total number of children 
undergoing painless procedural sedation was 170 patients, while it was 780 children for painful procedural sedation.

Median dose of triclofos during painless procedures was $44 \mathrm{mg} / \mathrm{kg}$ and it was $76.25 \mathrm{mg} / \mathrm{kg}$ during painful procedures.

14 studies met the inclusion criteria for analysis of AEs. Vomiting was the most commonly reported AE, incidence rate of 7.1 per 100 children. Most were not severe and did not warrant medical intervention.

The second most frequently reported $\mathrm{AE}$ was hypoxia, incidence rate of 6.9 per 100 children, and it was reported only in healthy children undergoing painful procedural sedation. All cases of hypoxia were mild (SpO2 90-95\%) and did not require medical intervention.

The incidence rate of reported AEs was dramatically higher for painful procedures compared to painless procedures $(14.1 \%$ versus $1.5 \%$ ) respectively. There were no deaths, however two serious AEs due to administration medication errors were reported, both required medical interventions and hospitalisation.

The success rate of painless procedural sedation compared to painful procedures (particularly dental) was found to be higher (ranged from $84 \%$ to $100 \%$ versus 38 to $95 \%$ ) respectively.

Conclusions The systematic review suggests that triclofos seems to have a good sedative effect mainly with short painless procedural sedation. Vomiting and hypoxia AES were the most commonly reported AEs. However, these findings are limited by patients' number and the lack of uniformity of outcome reporting systems. 